\title{
Importancia de la investigación en la formación de estudiantes en la modalidad a distancia*
}

\author{
José Eriberto Cifuentes Medina ${ }^{1}$ \\ (iD http://orcid.org/0000-0001-5702-620X \\ José Irenarco Pedraza Suaréz ${ }^{2}$ \\ (D) http://orcid.org/0000-0001-6797-852X \\ Universidad Pedagógica y Tecnológica de Colombia, Colombia
}

DOI: http://dx.doi.org/10.17081/eduhum.19.32.2530

Recibido: 30 de junio de 2016

Aceptado: 01 de octubre de 2016

\section{The importance of researching for technicians' training through distance learning}

Palabras clave:

Investigación, Formación,

Tecnólogos,

Regencia en Farmacia,

Gestión en Salud.

Keywords:

Research, Training,

Technologists,

Regency of Pharmacy,

Health Management.

\begin{abstract}
Resumen
El presente artículo diserta acerca del papel y la importancia de las asignaturas de "Introducción a la Investigación" y "Metodología de la Investigación" en la formación de tecnólogos, que se ofrece en la modalidad de estudios a distancia. En conclusión, se hace necesaria una reflexión acerca de las generalidades de investigación, normatividad para la presentación de trabajos e informes, y la elaboración, sustentación de proyectos y artículos de investigación como principios fundantes de la proyección en esencia y existencia de la investigación. Las asignaturas mencionadas hacen parte del proceso académico-investigativo y parten de lo que se ha observado en la realidad estudiantil, en los procesos académicos y en la experiencia docente; todo ello con el fin de concretar posibilidades de cambio, acción y transformación de su labor como regentes en farmacia y administradores del sistema de la salud, teniendo en cuenta las condiciones de la "Educación a distancia" en la que los estudiantes se forman de la mano del aprendizaje autónomo, pero también a la necesidad de acudir a la investigación desde la realidad y contexto que los rodea con bases teóricas consistentes y en aras de ejercer su profesión de una manera eficiente.
\end{abstract}

\begin{abstract}
This paper focuses on the distance learning educational process considering the role and importance of "Introduction to Research" and "Research Methodology" subjects in technologists' educational process. In conclusion, a reflection about research generalities, standards for the submission of papers and reports, and the development, sustainability of projects and research articles as foundational principles of the projection in essence and existence of the research are needed. The subjects mentioned above are part of the academic and research process and deduce from what has been observed in the real life as well as in academic processes and teaching experiences; this study was conducted in order to realize opportunities for changing, action, and transformation of its work as regents in pharmaceutical and system administrators of health. We must take into account the conditions of the "Distance education" in which students are trained through an autonomous learning, but also the need to research based on the reality and their context with theoretical basis consistent to exercise their profession in an efficient way.
\end{abstract}

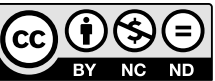

Referencia de este artículo (APA): Cifuentes, J. \& Pedraza, J. (2017). Importancia de la investigación en la formación de estudiantes en la modalidad a distancia. En Revista Educación y Humanismo, 19(32), 31-52. http://dx.doi.org/10.17081/eduhum.19.32.2530

* Artículo de Revisión de tema. Hace parte del proyecto: "Retos y desafíos de la investigación en la modalidad a distancia".

1. Estudiante de Doctorado en Educación, Universidad de Baja California, México. Magíster en Educación, Especialista en Educación con énfasis en Evaluación Educativa, Licenciado en Teología, Licenciado en Filosofía y Educación Religiosa, Universidad Santo Tomás, Colombia. Docente-investigador Universidad Pedagógica y Tecnológica de Colombia. joseeriberto.cifuentes@uptc.edu.co

2. Estudiante de Doctorado en Gerencia Pública y Política Social, Universidad de Baja California, México. Magíster en Administración, Especialista en Evaluación Pedagógica, Administrador de Empresas, Universidad Pedagógica y Tecnológica de Colombia. Director de la Escuela de Ciencias Administrativas y Económicas, Facultad de Estudios a Distancia de la Universidad Pedagógica y Tecnológica de Colombia. jose.pedraza@uptc.edu.co 


\section{Introducción}

En la formación de tecnólogos de Regencia en Farmacia y Gestión en Salud, la investigación es pertinente, tanto en su preparación académica para un desarrollo investigativo durante el curso académico como para su posterior desempeño laboral y profesional. Se pretende abordar, en consecuencia, algunas generalidades de la investigación, la legislación educativa, sus ventajas, la evaluación del aprendizaje, las normas de presentación de trabajos escritos (APA e ICONTEC) y algunos elementos necesarios para la elaboración, la presentación y la ejecución de proyectos de investigación.

El artículo toma como punto de referencia los programas de Tecnología en Regencia en Farmacia y Gestión en Salud, adscritos a la Escuela de Ciencias Administrativas y Económicas de la Facultad de Estudios a Distancia; concretamente, el proceso de enseñanza de las asignaturas de Introducción y Metodología de la Investigación, y la necesidad de demanda de docentes idóneos que orienten la investigación con espíritu crítico, analítico, argumentativo y propositivo, así como de estudiantes capaces de interrogarse por sí mismos desde su contexto social, y su realidad profesional y laboral.

Para el desarrollo de la investigación, se tuvieron en cuenta aspectos o tópicos de trabajo que contribuyen a la solidez teórica del artículo, a saber: modalidad de educación a distancia, aspectos administrativos de los procesos de investigación y pautas para la elaboración y ejecución de un proyecto de investigación. Además, de manera sucinta se señala la tipología de artículos considerada por Colciencias, y elementos de la escritura académica y científica en estudiantes de programas tecnológicos.

\section{Modalidad de educación abierta y a distancia}

La educación abierta y a distancia se puede definir como un sistema "en el que la educación se puede ofrecer en cualquier tiempo y en cualquier lugar, a través del uso de diversos medios de comunicación electrónica" (De la Torre, 2009, p.240). Esta modalidad educativa brinda beneficios y fortalece procesos de formación a la población que no puede acceder a la educación presencial. La educación a distancia también es definida por Corrales (2008) como:

Situaciones de enseñanza y aprendizaje en las que el docente o instructor y el alumno o estudiante están geográficamente separados $\mathrm{y}$, por consiguiente, se apoyan en materiales impresos u otro tipo de materiales electrónicos para la consecución del aprendizaje. La formación a distancia incluye, por tanto, la enseñanza a distancia - papel que corresponde al profesor o tutor-, y el aprendizaje a distancia -papel que corresponde al alumno-. (p.15)

La modalidad de educación a distancia surge, entonces, como respuesta a las necesidades de jóvenes, adultos y profesionales que no pueden asistir a un sistema escolarizado presencial. Los 
avances tecnológicos en materia de cómputo y telecomunicaciones ayudan a dar respuesta a dichas necesidades y se convierten en un apoyo para esta forma de educación que se puede ofrecer en cualquier tiempo y lugar, a través del uso de diversos medios de comunicación; todo ello teniendo en cuenta la diversidad de herramientas a las cuales permite acudir en el desarrollo de calidad de la educación.

Es importante reconocer que esta modalidad o sistema se puede utilizar en los diferentes niveles educativos (secundaria y superior), e impartir en instituciones públicas y privadas. Sin embargo, por las particularidades descritas, la educación a distancia es un reto y para que "funcione de manera óptima, se requiere una capacidad de autoaprendizaje en el alumno que, por lo general, en edades tempranas no posee, y debe adquirir durante la educación obligatoria" (De la Torre, 2009, p.237).

$\mathrm{La}$ formación tecnológica y profesional corresponde, por otra parte, al "nivel educativo conocido como formación profesional, vocacional o técnica, tiene por finalidad específica la educación integral y su capacitación para el ejercicio de una profesión específica" (De la Torre, 2009, p.239). Por lo tanto, la formación en un área tecnológica corresponde a la preparación o capacitación en áreas disciplinarias, en pro de la consecución de un título de auxiliar, técnico, tecnólogo o profesional, que habilita al sujeto para algo definido, en la medida que pueda ser competente en el saber y hacer, para lo cual contribuye esta modalidad de educación.

\section{Características de la educación a distancia}

Corrales (2008) resalta un número considerable de características como parte de una educación a distancia sólida, de calidad y al servicio de muchas generaciones. A continuación, se presentan a manera de síntesis:

Tabla 1. Características de la educación a distancia

\begin{tabular}{|c|c|c|}
\hline No. & Característica & Definición \\
\hline 1 & $\begin{array}{l}\text { Elimina la barrera } \\
\text { del espacio y de la } \\
\text { distancia }\end{array}$ & $\begin{array}{l}\text { El estudiante y el docente se en- } \\
\text { cuentran separados, pero siguen un } \\
\text { proceso de enseñanza-aprendizaje } \\
\text { desde la distancia, para el encuen- } \\
\text { tro solo pueden asistir quienes vi- } \\
\text { ven cerca. }\end{array}$ \\
\hline 2 & $\begin{array}{l}\text { Elimina la barrera } \\
\text { de la edad y de la } \\
\text { ocupación }\end{array}$ & $\begin{array}{l}\text { La necesidad de trabajar impedía } \\
\text { a personas adultas y jóvenes tener } \\
\text { acceso a la formación convencio- } \\
\text { nal. La metodología a distancia } \\
\text { permite estudiar a cualquier per- } \\
\text { sona que trabaje. El estudiante es } \\
\text { quien establece sus horarios de } \\
\text { estudio. }\end{array}$ \\
\hline 3 & $\begin{array}{l}\text { El grupo de } \\
\text { estudiantes es } \\
\text { heterogéneo }\end{array}$ & $\begin{array}{l}\text { No solo por las diferencias de } \\
\text { edad, sino por la diversidad de ocu- } \\
\text { paciones y la desigual preparación } \\
\text { que caracteriza sobre todo a los } \\
\text { alumnos de mayor edad. }\end{array}$ \\
\hline 4 & $\begin{array}{l}\text { Población } \\
\text { especialmente } \\
\text { dispersa }\end{array}$ & $\begin{array}{l}\text { Se vinculan alumnos de muchos } \\
\text { lugares, en un área más o menos } \\
\text { extensa según sea la cobertura o } \\
\text { radio de acción de los programas } \\
\text { que se ofrecen. No hay encuentros } \\
\text { presenciales diarios. }\end{array}$ \\
\hline 5 & $\begin{array}{l}\text { Permite el acceso } \\
\text { masivo }\end{array}$ & $\begin{array}{l}\text { Constituye un valioso aporte a la } \\
\text { democratización de la educación al } \\
\text { facilitar el acceso a sectores de la } \\
\text { población que no podían ingresar } \\
\text { en los cursos presenciales. }\end{array}$ \\
\hline 6 & $\begin{array}{l}\text { Facilita el trabajo } \\
\text { individual }\end{array}$ & $\begin{array}{l}\text { En cuanto al tiempo y la capacidad } \\
\text { de aprendizaje, reconoce que exis- } \\
\text { ten en las personas y los grupos di- } \\
\text { ferentes estilos cognitivos, ritmos } \\
\text { y condiciones de aprendizaje. }\end{array}$ \\
\hline
\end{tabular}


Continuación Tabla 1

\begin{tabular}{|c|l|l|}
\hline 7 & $\begin{array}{l}\text { El alumno es } \\
\text { el centro del } \\
\text { aprendizaje }\end{array}$ & $\begin{array}{l}\text { Es quien debe gestionar su propia } \\
\text { formación a través de procesos de } \\
\text { aprendizaje dotados de gran auto- } \\
\text { nomía y responsabilidad. }\end{array}$ \\
\hline 8 & $\begin{array}{l}\text { Variedad de medios } \\
\text { y materias }\end{array}$ & $\begin{array}{l}\text { Elaborados específicamente para } \\
\text { fomentar el autoaprendizaje: mate- } \\
\text { riales impresos y audiovisuales, de } \\
\text { comunicación, tutorías y asesorías } \\
\text { personales y grupales, etc. }\end{array}$ \\
\hline 9 & $\begin{array}{l}\text { Se mueve en } \\
\text { el horizonte de } \\
\text { la educación } \\
\text { permanente }\end{array}$ & $\begin{array}{l}\text { Los procesos formativos y de } \\
\text { construcción del conocimiento du- } \\
\text { ran toda la vida de la persona, se } \\
\text { pueden ofrecer los mismos progra- } \\
\text { mas que en la educación presencial } \\
\text { y otros orientados a la población } \\
\text { adulta, trabajadores y a otras per- } \\
\text { sonas que difícilmente podrían } \\
\text { acceder. }\end{array}$ \\
\hline
\end{tabular}

Fuente: Adaptado de Corrales (2008)

En la educación a distancia la formación se da entre el docente y el estudiante de forma independiente, sin precisión de lugar y tiempo, y de acuerdo con los diferentes ritmos y condiciones de comunicación e interacción, con el objeto de propiciar el aprendizaje. En este sentido, Basabe (2007) considera a la modalidad como una propuesta educativa revolucionaria, "consiste en el envío electrónico de contenidos curriculares fuera de la universidad con acceso del usuario en tiempos alternos. La imagen virtual del profesor con frecuencia es la portadora de contenidos" (p.22). Los docentes asumen un gran reto al diseñar nuevos materiales para interactuar en un nuevo escenario.

\section{Ventajas e inconvenientes en la educación a distancia}

La modalidad de educación a distancia fortalece el desarrollo investigativo en curso; no obstante, es pertinente abordar las posibles ventajas e inconvenientes desde la perspectiva de Corrales (2008), en la que se destacan los siguientes:

Tabla 2. Ventajas e inconvenientes de la modalidad a distancia

\begin{tabular}{|l|l|}
\hline \multicolumn{1}{|c|}{ Ventajas } & \multicolumn{1}{c|}{ Inconvenientes } \\
\hline $\begin{array}{l}\text { Se evita el desplazamiento, } \\
\text { el estudiante no está sujeto a } \\
\text { instituciones físicas para ser } \\
\text { partícipe del proceso de ense- }\end{array}$ & $\begin{array}{l}\text { La inversión inicial puede ser } \\
\text { grande en comparación con } \\
\text { otros tipos de formación. }\end{array}$ \\
\hline $\begin{array}{l}\text { ñanza-aprendizaje. } \\
\text { mento de estudio que más le } \\
\text { interese, sin necesidad de un } \\
\text { horario predeterminado. }\end{array}$ & $\begin{array}{l}\text { Puede existir menos contacto } \\
\text { curso. }\end{array}$ \\
\hline $\begin{array}{l}\text { El estudiante fija su ritmo de } \\
\text { trabajo, avanzando según sus } \\
\text { conocimientos y disponibili- } \\
\text { dad. }\end{array}$ & $\begin{array}{l}\text { La tecnología más adecuada } \\
\text { puede ser inaccesible por su } \\
\text { costo. }\end{array}$ \\
\hline $\begin{array}{l}\text { El estudiante recibe atención } \\
\text { individualizada y permanente } \\
\text { por parte del docente a lo largo } \\
\text { del proceso formativo. }\end{array}$ & $\begin{array}{l}\text { Es necesario un sistema de } \\
\text { apoyo, que pueda solucionar } \\
\text { cualquier duda o problema que } \\
\text { le surja al estudiante. }\end{array}$ \\
\hline $\begin{array}{l}\text { La formación llega a un mayor } \\
\text { número de estudiantes al mis- } \\
\text { mo tiempo, reduciendo esfuer- } \\
\text { zos y gastos. }\end{array}$ & $\begin{array}{l}\text { Se pueden encontrar proble- } \\
\text { mas a la hora de acceder a los } \\
\text { recursos disponibles en la red, } \\
\text { por diferentes causas: falta de } \\
\text { electricidad, falla en los orde- } \\
\text { nadores, etc. }\end{array}$ \\
\hline
\end{tabular}

Fuente: Adaptado de Corrales (2008)

En el escenario de la educación a distancia, predominan las ventajas sobre los inconvenientes. Como puede verse, estos últimos son más bien de logística y quizás de recursos físicos, que siempre se pueden solucionar. En la comunicación sobresale el poder de la palabra escrita, las ayudas audiovisuales y demás elementos propios de comunicación como el teléfono y el internet, al igual que el apoyo de las plataformas virtuales con sus herramientas. 
La modalidad a distancia de la Universidad Pedagógica y Tecnológica de Colombia tiene como punto de partida el año de 1983, cuando se crea el Instituto de Educación a Distancia con vigencia de 19 años. En el año 2002, se transforma la Facultad en la forma que subsiste hasta la actualidad. Hoy día, la educación a distancia en esta universidad se apoya en la plataforma Moodle, a través de internet y las diferentes herramientas que ofrecen las Tecnologías de la Información y la Comunicación (TIC), lo cual conlleva a las ventajas presentadas en la Tabla 3.

Tabla 3. Ventajas del uso de tecnologías de información y comunicaciones en educación

\section{VENTAJAS DEL USO DE TIC}

- Incorpora las tecnologías de la información y la comunicación, como elemento diferencial.

- La comunicación es interactiva, allí los alumnos pueden comunicarse unos con otros, con el formador y con los recursos on-line disponibles.

- La variedad de instrumentos está combinada con: materiales de estudio; $\mathrm{CD}$, videos, materiales en redes y la comunicación sincrónica (chat) y asincrónica (correo electrónico, listas de discusión, foro, entre otros).

- El estudiante controla su aprendizaje, lo que aprende y el orden en que lo aprende, desde la perspectiva de su autonomía.

- Cursar una asignatura a través de la plataforma implica fortalecer el aprendizaje autónomo, ya que se puede desarrollar completamente on-line contenidos, actividades y evaluación.

- Los estudiantes pueden cursar una asignatura a través de la plataforma independientemente de la ubicación geográfica.

- Se favorece el aprendizaje colaborativo, la discusión, intercambio de ideas, para realizar las actividades del curso.

- La formación on-line ofrece la posibilidad de evaluar también on-line a los estudiantes, a través de test incorporados en el programa.

- Ofrece la opción a cada estudiante de conocer el seguimiento personalizado de su asignatura y de sus calificaciones.

Fuente: Adaptado de Corrales (2008)

Se han conjugado las teorías de Corrales (2008), De la Torre (2009) y Basabe (2007) en torno a la modalidad de educación a distancia, como una posibilidad de formación a la que muchos sectores de la población tienen acceso sin dejar de trabajar y atender otros quehaceres de la cotidianidad. De este modo, la modalidad se constituye en una experiencia que contribuye a la superación de los seres humanos en pro de un desarrollo justo y equitativo para el progreso de la sociedad.

\section{Generalidades de la investigación}

En el desarrollo de esta segunda categoría de estudio en el presente artículo, es significativo dar inicio realizando algunas preguntas como: ¿Qué es la investigación?, ¿por qué investigar?, ¿cuándo investigar? y ¿cómo investigar?, a fin de despertar la curiosidad y la necesidad de formar tecnólogos desde y para el contexto, a la luz de los aspectos de vanguardia en procesos de investigación.

En los principios epistemológicos, resulta pertinente la aproximación a la investigación desde la etimología misma de la palabra que la designa y desde diversos teóricos. El término viene de las raíces latinas investigium - ire, que significa ir tras la huella, en otras palabras, se trata de un camino que se recorre en búsqueda de respuestas (Castillo, 2004, p.11). La precisión original es ineludible para conocer otras posturas de lo que es y puede ser.

En este sentido, Munch (2009) afirma: "Se puede asumir la investigación como aquel proceso que se desarrolla con el fin de lograr 
conocimientos nuevos, generalmente orientados -en el corto o en el largo plazo- a la solución de problemas o a la satisfacción de necesidades" (p.25). En consecuencia, se puede considerar la investigación como un proceso sistemático, organizado y progresivo con el fin de dar respuesta a los interrogantes que surgen en el contexto académico, laboral o profesional; y responder de manera objetiva y clara a un tejido de la realidad como punto de partida.

El citado Munch (2009) también plantea que la investigación significa: "averiguar, indagar, buscar" y puede considerarla como "una actividad humana inherente a la naturaleza humana, aunque no por esto se pretenda afirmar que todos los seres humanos sean investigadores" (p.25). Por tanto, se puede razonar desde una investigación cotidiana hasta la de carácter científico. Esta última constituye un carácter riguroso y exacto, de acuerdo con un proceso y método, con el fin de darle confiabilidad y validez, a través de las evidencias necesarias según el ángulo de investigación (cualitativa o cuantitativa) para su desarrollo y proceso.

Álvarez (1983) sostiene que la ciencia requiere de la investigación y que: "se puede entender tanto un proceso como un resultado. En cuanto proceso, la ciencia es la aplicación del método científico al conocimiento de las estructuras de alguna región de la realidad. En cuanto resultado, la ciencia es un conjunto de conocimientos racionales, sistemáticos, controlados y fiables (p.8).
Conviene comprender también que la investigación requiere seguir un camino, que en la ciencia se denomina método científico, y que en sí es un método para considerar una estructura. De acuerdo con los enfoques y tipos, cualitativo o cuantitativo, se sigue un camino o se desarrolla un método: la "Investigación exploratoria, explicativa, descriptiva, experimental, acción participativa-IAP, o etnográfica" (Castillo 2004, p.14).

Los enfoques, tipos, método y nociones generales de investigación ayudan en los procesos de este tipo a los tecnólogos en formación de Regencia en Farmacia y Gestión en Salud; y de forma concreta, facilitan a los estudiantes la realización de investigaciones de manera más práctica y eficiente.

\section{Ventajas de la enseñanza de la investi-} gación

La enseñanza-aprendizaje de la investigación con dedicación y compromiso de la teoría y la praxis conllevan una serie de ventajas para los futuros tecnólogos, entre otras, las siguientes:

1. El programa de Gestión en Salud considera el tema en dos semestres: en IV semestre, con la asignatura Introducción a la Investigación; y en $\mathrm{V}$, con Metodología de la Investigación. En el primer caso se consolidan las bases y soportes epistemológicos y metodológicos; $\mathrm{y}$ en el segundo se profundiza en aspectos teóricos, pero se centra en la praxis. Son elementos que permiten la diferencia con el programa de Regencia, que se orienta solo en Metodología de la Investigación y se ha de aprovechar en los aspectos conceptuales y prácticos de la investigación. 
2. En cada programa se tienen en cuenta sus particularidades, ya que en el caso de Gestión en Salud se dirige a la administración; y en Regencia en el manejo, distribución, supervisión, vigilancia de medicamentos y como auxiliares del químico farmacéutico.

3. La enseñanza de la investigación permite a los estudiantes manejar tanto la conceptualización de investigación de diferentes formas y con diversos ejercicios, para luego llevarlos a la práctica en la elaboración, la ejecución y la presentación de proyectos de investigación desde su realidad y contexto; en aras de adelantar a futuro procesos rigurosos de investigación y la escritura científica.

4. Como parte de la comprensión y estrategia se trabajan los relatos y películas de Sherlock Holmes (Pineda, 2008), a fin de construir rutas viables y posibles para la disolución de las dificultades de la vida cotidiana, todo ello en desarrollo del propósito de un alistamiento a la solución de problemas complejos $\mathrm{y}$ con la rigurosidad que se puede aplicar a los procesos científicos.

5. Se prepara a los tecnólogos en formación en la elaboración, la formulación, la ejecución y la presentación de proyectos de carácter investigativo desde y para su realidad, en el propósito de teorizarlos y adelantar vías posibles de solución desde el método científico. Tal será el objetivo culmen de la investigación, que desde la universidad apenas es formativa.

6. Despierta en el estudiante la curiosidad y el asombro al igual que "una capacidad para la contemplación y el trabajo reflexivo" (Pineda, 2011, p.45), en la medida en que el asombro provoque un conocimiento novedoso $\mathrm{y}$ propicie el cultivo de hábitos científicos e investigativos.

Aparte de las ventajas en mención, existen otras que los docentes y estudiantes han de ir descubriendo en el proceso de la enseñanzaaprendizaje, pues solo con la experiencia se fortalecen verdaderos procesos de investigación, que emergen del quehacer académico, profesional y laboral.

\section{Marco normativo acerca de la investi-} gación en programas de carácter tecnológico

Es necesario considerar como parte del estatus epistemológico y pragmático, la normativa en relación a leyes y decretos, que de manera directa o indirecta refieren a la investigación en la educación superior en los programas tecnológicos.

Tabla 4. Legislación nacional e institucional

\begin{tabular}{|l|l|}
\hline \multicolumn{1}{|c|}{$\begin{array}{c}\text { DECRETO Y/O } \\
\text { LEY }\end{array}$} & \multicolumn{1}{c|}{ CONTENIDO } \\
\hline $\begin{array}{l}\text { Decreto 2667 de 17 } \\
\text { de diciembre de 1976 }\end{array}$ & $\begin{array}{l}\text { Por el cual se reglamentan las carreras } \\
\text { tecnológicas. }\end{array}$ \\
\hline $\begin{array}{l}\text { Ley } 30 \text { de } 29 \text { de } \\
\text { diciembre de } 1992\end{array}$ & $\begin{array}{l}\text { Por la cual se organiza el servicio públi- } \\
\text { co de la educación superior. }\end{array}$ \\
\hline $\begin{array}{l}\text { Ley } 749 \text { de } 19 \\
\text { de julio de } 2002\end{array}$ & $\begin{array}{l}\text { Por la cual se organiza el servicio públi- } \\
\text { co de la educación superior en las moda- } \\
\text { lidades de formación técnica profesional } \\
\text { y tecnológica. }\end{array}$ \\
\hline $\begin{array}{l}\text { Decreto } 1295 \text { de } \\
20 \text { de abril de 2010 }\end{array}$ & $\begin{array}{l}\text { Por el cual se reglamenta el registro ca- } \\
\text { lificado de que trata la Ley 1188 de 2008 } \\
\text { y la oferta y desarrollo de programas } \\
\text { académicos de educación superior. }\end{array}$ \\
\hline
\end{tabular}




Continuación Tabla 4
\begin{tabular}{|l|l|}
\hline Resolución No. 36 & Por la cual se adoptan y reglamentan las \\
de 2010 a nivel insti- & modalidades de trabajo de grado para los \\
tucional y propio de & estudiantes de los programas de pregra- \\
la Facultad & $\begin{array}{l}\text { do ofrecidos por la Facultad de Estudios } \\
\text { a Distancia de la Universidad Pedagógi- } \\
\text { ca Tecnológica de Colombia. }\end{array}$ \\
\hline
\end{tabular}
Fuente: Elaboración propia

El marco normativo señalado en la Tabla 4 permite evidenciar en parte la legislación que reglamenta las carreras de educación superior en las modalidades de formación técnica y tecnológica, en las que están inmersos los programas estudiados en este artículo.

Al interior de la universidad, disposiciones como la Resolución No. 40 de 2008, Por la cual se reglamenta el área general, modificada por la Resolución No. 58 de 2009; así como el Acuerdo No. 050 de 2008, Por el cual se establecen los criterios para la implementación del Sistema de Créditos y se definen las Áreas de Estructuración Curricular de los Programas de Pregrado Presenciales, en la Universidad Pedagógica y Tecnológica de Colombia, modificado por el Acuerdo No. 086 de 2009 , evidencian que la normatividad nacional también se ha aplicado a nivel institucional en las diversas reformas curriculares planteadas en aras de consolidar los principios de la investigación formativa y su proyección a la investigación propiamente dicha.

La normatividad permite evidenciar la creación de los programas a los cuales se ha hecho referencia, así como reconocer su trayectoria, tal como indican, por ejemplo, el Acuerdo 072 del 15 de julio de 1998; Por el cual el Consejo Superior de la UPTC, crea el Programa de Gestión en Salud en la modalidad de Educación a Distancia; y el Acuerdo No. 0116 de fecha 17 de noviembre de 1998; Por el cual el Consejo Superior creó el programa de Tecnología en Regencia en Farmacia, en la modalidad de Educación a Distancia. Así, a lo largo de casi dos décadas se han estado formando tecnólogos en las direcciones mencionadas según las competencias establecidas en el perfil profesional y laboral. Más concretamente, en relación a la investigación formativa y la proyección científica, en los últimos años y mediante las diversas prácticas, se ha consolidado el desarrollo de laboratorios según aspectos referidos a la praxis que tienen como punto de partida el proceso de enseñanza-aprendizaje desde el aula de clase, y la interacción a través del aula virtual con las diferentes herramientas que permiten la interrelación permanente, consecuente y consistente en y para los procesos de investigación.

En la Universidad Pedagógica y Tecnológica de Colombia, que presenta las dos modalidades de formación en pregrado, presencial y a distancia, se han consolidado, entonces, los procesos de investigación formativa y su proyección a la investigación aplicada. Sin embargo, se reconoce que en la modalidad a distancia las condiciones y características son particulares; por ello, el Consejo Académico, a través de la Resolución 036 del 18 de mayo de 2010 especifica en el artículo $\mathrm{N}^{\circ}$ 2: Establecer las modalidades de Trabajo de Grado para los Programas de Pregrado 
de la Facultad de Estudios a Distancia, así: Para Programas Tecnológicos Profesionales: 1. Participación activa en un Grupo de Investigación Institucionalmente reconocido por la DIN. 2. Práctica con Proyección Empresarial o Social y 3. Participación en Diplomados.
Para alcanzar el grado de técnicos profesionales solo se evidencia la práctica que en relación a la investigación contiene la estructura metodológica de un proyecto de investigación. Para la obtención del título de tecnólogos, entre

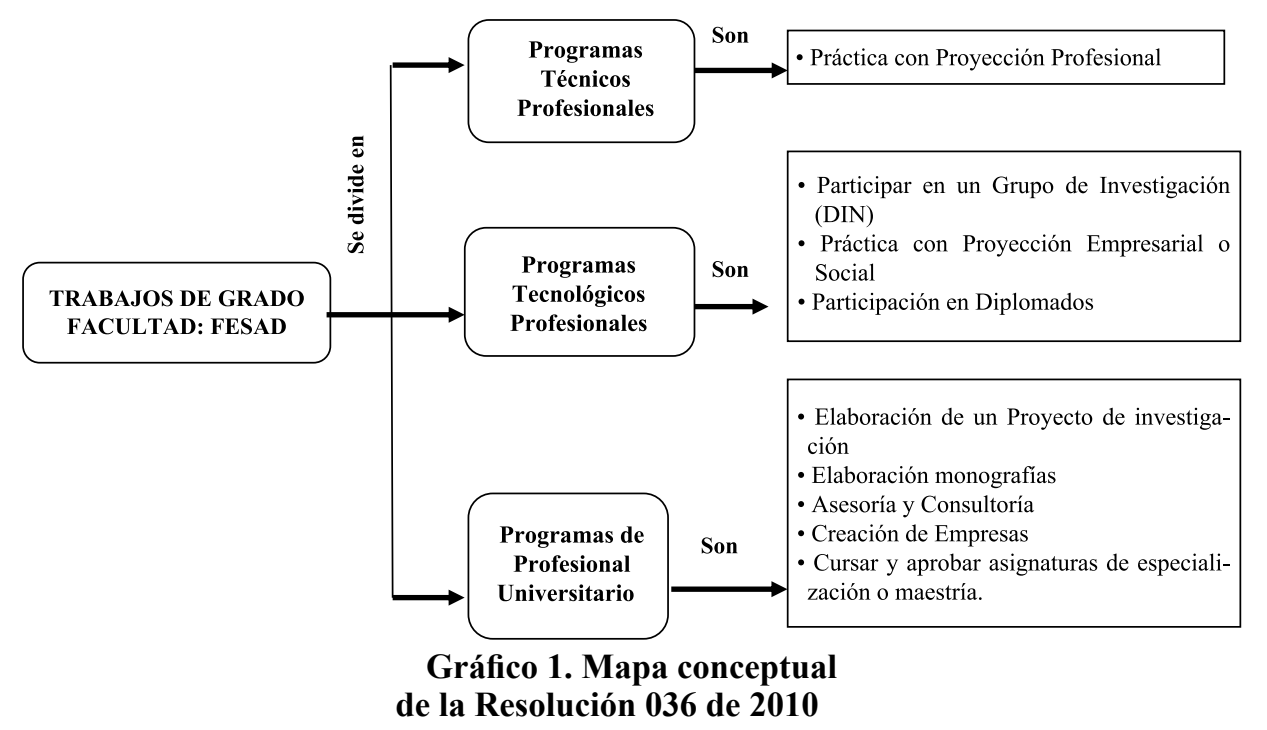

La Resolución que rige para la modalidad a distancia permite como opción de grado la participación de manera activa en grupos de investigación reconocidos por la Dirección de Investigaciones (DIN). En realidad el número de estudiantes que se acogen a esta posibilidad son escasos, pero a la fecha no se ha adelantado un estudio que considere la falta de interés, desconocimiento o las razones de asumir al cumplimento de los requisitos de grado mediante esta posibilidad. El compromiso de hacerla realidad ha de incluir a las directivas de Facultad, la Escuela y docentes; no solo para orientar, motivar e incentivar a los estudiantes para que escojan la iniciativa en mención no solo como opción de grado, sino como un propósito que los constituya en verdaderos investigadores. las tres posibilidades existentes, la participación en diplomados ocupa el primer lugar (75\% aproximadamente) por su facilidad en la oferta virtual; mientras que la práctica con proyección empresarial ocupa el segundo con un $24 \%$; y la participación en grupos de investigación, apenas cuenta con un simple $1 \%$. Son consideraciones a la luz de la experiencia sin la pericia de un estudio consistente, pero que demuestra la evidente necesidad de formular políticas estratégicas para la promoción y participación de los estudiantes en semilleros, proyectos y grupos de investigación.

Por otra parte, la universidad, en su Unidad de Política Social, proyecta la oferta de becas por 
diversos aspectos y propone entre ellos becas por investigación, respecto a lo cual se ha obtenido información de 2010 a 2012, lapso de tres años en el que solo seis de los más de mil estudiantes que han participado en esta oferta de becas han optado por la investigación. Al respecto se debe tomar en cuenta que en tres años se cursa la carrera de los dos programas en referencia, dado que comprenden un plan de estudios de seis semestres. no solo pueden deberse a las particularidades de tiempo y espacio que dinamizan la Facultad de Estudios a Distancia.

\section{Planes de estudio de los programas Gestión en Salud y Regencia en Farmacia}

De acuerdo con la reglamentación de la universidad respecto a créditos y asignaturas, es necesario que se organice el plan de estudios de los programas de las carreras tecnológicas en referencia.

Tabla 5. Estudiantes beneficiarios de becas por parte de Bienestar Universitario de la UPTC

\begin{tabular}{|c|c|c|c|c|c|c|c|}
\hline \multirow{2}{*}{$\begin{array}{c}\text { Facultad } \\
\text { de estudios } \\
\text { a distancia }\end{array}$} & $\begin{array}{c}\text { Beca } \\
\text { Trabajo }\end{array}$ & $\begin{array}{c}\text { Beca } \\
\text { Alimentación }\end{array}$ & $\begin{array}{c}\text { Beca } \\
\text { Investigación }\end{array}$ & $\begin{array}{c}\text { Beca Extrema } \\
\text { Incapacidad } \\
\text { Económica }\end{array}$ & $\begin{array}{c}\text { Becas Convenio } \\
\text { Gobernación }\end{array}$ & $\begin{array}{c}\text { Beca por Representación } \\
\text { Cultural y/o } \\
\text { Deportiva }\end{array}$ & Total \\
\hline I de 2010 & 3 & 0 & 2 & 12 & 0 & 1 & 5 \\
\hline II de 2010 & 9 & 0 & 2 & 15 & 0 & 0 & 31 \\
\hline I de 2011 & 17 & 0 & 0 & 13 & 0 & 0 & 30 \\
\hline II de 2011 & 14 & 0 & 1 & 8 & 0 & 0 & 23 \\
\hline I de 2012 & 16 & 0 & 0 & 17 & 0 & 0 & 333 \\
\hline II de 2012 & 12 & 0 & 1 & 9 & 0 & & 22 \\
\hline
\end{tabular}

Fuente: Elaboración propia

La mayor parte de becas se justifican entonces en el trabajo y la extrema incapacidad económica, pero respecto a la investigación la intención es mínima y en vez de aumentar ha disminuido; por lo tanto, es necesario e importante seguir formando, preparando y motivando a los estudiantes en proceso de investigación con el fin de acceder a estos beneficios que ofrece la universidad y para fortalecer la importancia de la investigación en la formación de tecnólogos, aun cuando un objeto de otro estudio investigativo podría consistir en ampliar el rango y periodo de estudio al igual que las causas de la no aplicación a las becas por investigación, que
Tabla 6. Áreas y componentes de formación en Tecnología en Regencia en Farmacia

\begin{tabular}{|c|c|c|}
\hline Área & Créditos & Porcentaje \\
\hline General & 12 & $12 \%$ \\
\hline Interdisciplinar & 26 & $27 \%$ \\
\hline Disciplinar y profundización & 58 & $61 \%$ \\
\hline Total & 96 & $100 \%$ \\
\hline
\end{tabular}

Fuente: Elaboración propia

En este sentido, la Tabla 6 nos muestra la distribución de las materias según los créditos y el porcentaje en cada área en el plan de estudios. La investigación está ubicada dentro del área interdisciplinar, en la que hay 26 créditos y el $27 \%$ de espacio en la malla curricular. Dicha Tabla refiere a Regencia en Farmacia, en tanto 
la Tabla 7, a la Tecnología en Gestión en Salud; ambas evidencian en proyección comparativa las modificaciones en los créditos y porcentajes que existen entre carreras tecnológicas.

Tabla 7. Áreas y componentes de formación en Gestión en Salud

\begin{tabular}{|c|c|c|}
\hline Área & Créditos & Porcentaje \\
\hline General & 9 & $10 \%$ \\
\hline Interdisciplinar & 28 & $30 \%$ \\
\hline Disciplinar y profundización & 56 & $60 \%$ \\
\hline TOTAL & 96 & $100 \%$ \\
\hline
\end{tabular}

Fuente: Elaboración propia

El programa de Tecnología en Gestión en Salud y el programa de Tecnología en Regencia en Farmacia se crearon con los debidos soportes legales a nivel nacional e institucional, y hacen hincapié en la importancia de la investigación en la formación de tecnólogos, pero, obviamente, no han tenido el suficiente impacto.

La investigación requiere ser enseñada por parte de profesores que hayan desarrollado habilidades y competencias investigativas, que no solo se asombren de la realidad, sino que además propongan alternativas de solución.

En la perspectiva de la Unesco en la conferencia celebrada en 2009 en París y según afirma Ravelo (2012), "la educación superior es un bien público, de responsabilidad de todos los países, y que es la base de la investigación, innovación y creatividad" (p.19). Además, la educación superior, nivel en cual se encuentran en formación los tecnólogos en referencia, es la plataforma apropiada continua diciendo el mismo autor para:

proporcionar competencias sólidas y pertinentes para el mundo presente y futuro, y debe contribuir a la educación de ciudadanos éticos, comprometidos con la construcción de la paz, la defensa de los derechos humanos y los valores de la democracia. De igual manera, en dicha Conferencia, se resaltó que la autonomía es requisito indispensable para lograr procesos de enseñanza con calidad, pertinencia, eficacia, transparencia y responsabilidad social. (p.20)

Por esta razón, los docentes que orientan la investigación y de manera especial en las carreras tecnológicas, han de contextualizar la realidad hasta proponer alternativas de solución por el camino de la investigación, la elaboración, la formulación, la ejecución y la presentación de proyectos, retando a los estudiantes a ser críticos, analíticos, reflexivos, y a que indaguen en la búsqueda de alternativas viables para responderle al entorno académico y laboral desde una investigación formativa y en la dirección de la investigación aplicada y científica.

Es claro que los docentes interactúan con los estudiantes en procesos de investigación de acuerdo con la organización académica-administrativa de los planes de estudios en donde tiene espacio la investigación como asignatura, y en este caso de las carreras tecnológicas en estudio según se evidencia en las Tablas 8 y 9. 
Tabla 8. Cuarto semestre de Gestión en Salud

\begin{tabular}{|c|c|c|}
\hline Asignatura & Créditos & Tipo \\
\hline Introducción a la Investigación & 2 & $\mathrm{~T}$ \\
\hline Administración de Personal & 3 & $\mathrm{~T}$ \\
\hline Sistemas de Información en Salud & 2 & $\mathrm{~T}$ \\
\hline Epidemiología & 3 & $\mathrm{~T}$ \\
\hline Electiva disciplinar: & 3 & $\mathrm{TP}$ \\
\hline Salud Ocupacional & 3 & $\mathrm{TP}$ \\
\hline TOTAL DE CRÉDITOS & 16 & \\
\hline
\end{tabular}

Fuente: Elaboración propia

Tomado del Plan de estudios del programa de la Tecnología en Gestión en Salud, adscrito a la Facultad de Estudios a Distancia de la Universidad Pedagógica y Tecnológica de Colombia

Como puede verse, las Tablas 8 y 9 refieren al plan de estudios del programa de Gestión en Salud, y en ellas se implementa la investigación en los semestres cuarto y quinto de un total de seis semestres académicos. En el diseño de este plan de estudios se pensó en la importancia de la investigación, de modo que en un espacio reducido de tres años los tecnólogos en formación tuviesen la oportunidad de acceder a la teoría y la praxis de la investigación.

Tabla 9. Quinto semestre de Gestión en Salud

\begin{tabular}{|l|c|c|}
\hline \multicolumn{1}{|c|}{ Asignatura } & Créditos & Tipo \\
\hline Metodología de la Investigación & 3 & TP \\
\hline Salud Pública & 3 & T \\
\hline Sistema de Garantía de Calidad & 3 & T \\
\hline Costos en Salud & 2 & TP \\
\hline Facturación en Salud & 3 & TP \\
\hline Psicología Organizacional & 2 & T \\
\hline TOTAL DE CRÉDITOS & 16 & \\
\hline
\end{tabular}

Fuente: Elaboración propia

Tomado del Plan de estudios del programa de la Tecnología en Gestión en Salud, adscrito a la Facultad de Estudios a Distancia de la Universidad Pedagógica y Tecnológica de Colombia

De manera específica en la Tecnología en Gestión en Salud, aparece la Introducción a la Investigación como asignatura en el cuarto semestre, con una intensidad de dos créditos; así como Metodología de la Investigación en quinto semestre con tres créditos. En cuarto semestre la asignatura es de tipo teórica, pero en quinto semestre es de carácter teórico-práctica, lo que implica una mayor responsabilidad en el desarrollo de sus contenidos programáticos, llevados a la praxis desde el contexto como punto de partida.

En la Tecnología de Regencia en Farmacia, la investigación se oferta en las mismas condiciones que en el programa de Gestión en Salud, tanto en tiempo, asignaturas y créditos; quienes diseñaron el plan de estudios, proyectaron así la investigación como única asignatura en quinto semestre, según se evidencia en la Tabla 10.

Tabla 10. Quinto semestre de Regencia en Farmacia

\begin{tabular}{|l|c|c|}
\hline \multicolumn{1}{|c|}{ Asignatura } & Créditos & Tipo \\
\hline Metodología de la Investigación & 2 & $\mathrm{~T}$ \\
\hline Farmacia Veterinaria & 3 & $\mathrm{TP}$ \\
\hline Toxicología & 3 & $\mathrm{~T}$ \\
\hline Farmacognosia & 2 & $\mathrm{~T}$ \\
\hline Farmacovigilancia & 3 & $\mathrm{~T}$ \\
\hline Electiva disciplinar: Salud Ocupacional & 3 & $\mathrm{TP}$ \\
\hline TOTAL DE CRÉDITOS & 16 & \\
\hline
\end{tabular}

Fuente: Elaboración propia

Tomado del Plan de estudios del programa de la Tecnología en Regencia en Farmacia, adscrito a la Facultad de Estudios a Distancia de la Universidad Pedagógica y Tecnológica de Colombia

En la Tecnología de Regencia en Farmacia, la asignatura de Metodología de la Investigación es teórica y con proyección hacia la praxis. Ello con el fin de ahondar en el ejercicio investigativo tanto en lo teórico como en lo práctico. No obstante, se requiere multiplicar el tiempo para trabajar los dos campos y que los estudiantes 
además de la reflexión crítica, analítica y reflexiva, formulen proyectos de investigación desde las realidades, contextos y espacios. En este marco, queda casi en el vacio la posibilidad de promover la escritura académica y científica derivada de la literatura revisada y de la sistematización de los resultados investigativos.

La modalidad de Educación a Distancia, con sus particularidades, características, problemas, ventajas y demás aspectos propios de la dinámica de desarrollo comprende varios aspectos de la conferencia de la Unesco en 2009, por ejemplo:

El aprendizaje abierto y a distancia y el uso de las TIC ofrecen oportunidades de ampliar el acceso a la educación de calidad. Los establecimientos de educación superior deben invertir en la capacitación del personal docente y administrativo para desempeñar nuevas funciones en sistemas de enseñanza y aprendizaje que se transforman. La ampliación del acceso plantea un desafío a la calidad de la educación superior (Ravelo, 2012, p.20).

La calidad y pertinencia en la educación superior en la modalidad a distancia se constituyen en ejes fundamentales para la formación de bases sólidas en la investigación formativa y en la proyección de indagación científica, es decir, se hace necesario fortalecer la formación investigativa en y desde la formulación de proyectos por parte de los estudiantes de carreras en el nivel de tecnologías.

\section{La investigación formativa y su proyección} a la aplicación

La investigación en las carreras tecnológicas es evidentemente formativa por las condiciones de tiempo y espacio de los programas y la modalidad, pero ello no escatima los esfuerzos necesarios para traspasar los escenarios que conducen de la teoría a la práctica. Por lo tanto, todo aquello que se pueda forjar en los educandos por el bien de la ciencia nunca será inútil. Sin que esto conlleve a excesos, esto debe ser visto como una gran oportunidad para proyectar a los estudiantes hacia la investigación.

\section{Proyección de posible guía en los proyectos de investigación}

En la labor docente de la Universidad Pedagógica y Tecnológica de Colombia se proyectó una guía instruccional para los estudiantes, con el fin de formular, ejecutar y presentar proyectos de investigación.

Los diferentes libros de metodología de la investigación presentan al respecto una diversidad de modelos a seguir, pero hace falta uno más sencillo y explicado para los estudiantes, pues se hace necesario un proceso inductivo de lo particular a lo general. La guía trae consigo beneficios para los alumnos en lo que corresponde a la investigación en la formación de tecnólogos en Regencia en Farmacia y Gestión en Salud. Con esta orientación se pretende iniciarlos en procesos investigativos, tanto en la formación académica como en la formación profesional y laboral. 
De manera general y teniendo en cuenta el punto de vista de varios autores, se toma como base a Castillo (2004) y se propone la guía básica para que los tecnólogos en formación de
Regencia y Gestión en Salud puedan formular, presentar y ejecutar proyectos de investigación (Tabla 11).

Tabla 11. Guía básica para la elaboración y presentación de proyectos de investigación

\begin{tabular}{|c|c|}
\hline Pasos & Características \\
\hline Título & $\begin{array}{l}\text { Es el resumen breve del proyecto, proporciona una idea global de lo que se va a hacer. Debe permitir la comprensión } \\
\text { del proyecto, siendo claro y preciso, reflejando su contenido. }\end{array}$ \\
\hline Introducción & $\begin{array}{l}\text { Tiene como fin plantear el tema, presentar la hipótesis y comunicar al lector los objetivos y métodos utilizados en el } \\
\text { desarrollo del problema de investigación. }\end{array}$ \\
\hline $\begin{array}{l}\text { Planteamiento } \\
\text { del problema }\end{array}$ & $\begin{array}{l}\text { Establece la dirección del estudio para lograr ciertos objetivos. En él se concreta el punto de partida y el punto de llegada } \\
\text { de la investigación; señalando de qué situación inicia su trabajo al investigador y dónde desea estar cuando termine } \\
\text { el proyecto. Debe quedar bien definido, delimitado y claro, para lo cual se debe analizar la viabilidad de adelantar } \\
\text { la investigación en el momento y bajo qué condiciones de tiempo, personal, recursos físicos y económicos se va a } \\
\text { emprender. }\end{array}$ \\
\hline Hipótesis & $\begin{array}{l}\text { Basadas generalmente en el conocimiento, la experiencia y la información obtenida. Su verificación se hace una vez se } \\
\text { tengan los resultados de la investigación propuesta. Son la guía o directriz que orienta el trabajo investigativo. } \\
\text { Es parte importante en el proceso de la evaluación del proyecto por parte de terceros, mediante ella(s) el evaluador } \\
\text { se percata de si el investigador sabe lo que está buscando y si el trabajo está encaminado hacia la dirección indicada. }\end{array}$ \\
\hline Objetivos & $\begin{array}{l}\text { Son enunciados claros y precisos de las pretensiones que se buscan. Delimitan el problema y permiten pasar del estado } \\
\text { actual al estado deseado del mismo. Facilitan la estructuración de la metodología, estableciendo los caminos de acción } \\
\text { y direccionan la investigación. Se les considera como los compromisos que adquieren los responsables del proyecto. } \\
\text { Por lo tanto, los objetivos deben cumplir con características de factibilidad, verificabilidad, precisión y concreción. Toda } \\
\text { investigación se evalúa por el logro de objetivos. }\end{array}$ \\
\hline $\begin{array}{l}\text { Objetivo } \\
\text { general }\end{array}$ & Representa el propósito final del proyecto, para su logro es necesaria la formulación de objetivos específicos. \\
\hline $\begin{array}{l}\text { Objetivos } \\
\text { específicos }\end{array}$ & $\begin{array}{l}\text { Se originan del objetivo general, indican lo que se pretende realizar en cada una de las etapas de investigación. Estos } \\
\text { deben ser evaluados en cada paso para conocer los distintos niveles de los resultados, no deben ser numerosos, es } \\
\text { conveniente que sean de dos a tres. }\end{array}$ \\
\hline Justificación & $\begin{array}{l}\text { Es la fundamentación de la importancia del problema que aborda el proyecto y la necesidad de realizar la investigación } \\
\text { para hallar su solución. } \\
\text { Son las razones que ameritan la concretización del proyecto y es importante tener en cuenta elementos como: grado de } \\
\text { pertinencia de la investigación; el tipo de articulación con políticas a todo nivel; la motivación del investigador para } \\
\text { realizarlo; la relevancia para la disciplina o disciplinas que confluyen en el proyecto y demás razones que se requieran } \\
\text { para sustentar al proyecto. }\end{array}$ \\
\hline Antecedentes & $\begin{array}{l}\text { En ellos se trata de hacer una síntesis conceptual de las investigaciones o trabajos realizados sobre el problema } \\
\text { formulado, para determinar el enfoque metodológico de la investigación. Se busca aprovechar las teorías existentes } \\
\text { sobre el problema y ser un medio seguro para lograr sus objetivos. } \\
\text { Nos permite evitar la duplicidad de esfuerzos, al facilitar el estado del conocimiento disponible para generar uno } \\
\text { nuevo, ahorrando así recorridos innecesarios. En la búsqueda y recopilación de antecedentes se hace necesario ser muy } \\
\text { selectivos en la revisión bibliográfica, consultando la más relevante y reciente. } \\
\text { Es importante enriquecer la búsqueda de antecedentes para contar con más elementos de referencia y de soporte para } \\
\text { que el investigador maneje el tema con más propiedad. } \\
\text { Entre las fuentes más importantes para los antecedentes tenemos: artículos publicados, informes finales de investigación, } \\
\text { trabajos de grado, memorias de eventos académicos, páginas especializadas del Internet, antecedentes de otros } \\
\text { proyectos, asesoría de investigadores, lo que se va obteniendo a través de la investigación. }\end{array}$ \\
\hline
\end{tabular}


Continuación Tabla 11

\begin{tabular}{|c|c|}
\hline $\begin{array}{l}\text { reo de } \\
\text { erencia }\end{array}$ & $\begin{array}{l}\text { s el conjunto de conocimientos e imágenes con significado que poseemos en nuestra conciencia y de los que nos } \\
\text { xiliamos para identificar la temática seleccionada. }\end{array}$ \\
\hline nceptual & $\begin{array}{l}\text { Todo problema nos lleva a la definición de conceptos, estos deben ser definidos dándoles el significado general que } \\
\text { se intenta dar a conocer. En este sentido, es necesario unir el estudio a la teoría, las definiciones operacionales son } \\
\text { esenciales para llevar a cabo cualquier investigación, porque los datos deben ser recogidos en términos de hechos } \\
\text { observables. }\end{array}$ \\
\hline frico & $\begin{array}{l}\text { Nos ayuda a precisar y organizar los elementos contenidos en la descripción del problema, de tal manera que pueden ser } \\
\text { manejados y convertidos en acciones concretas. Se refiere al conocimiento básico y especializado, existente en torno } \\
\text { al tema del proyecto. En él se exponen teorías, principios y enfoques que brindan aportes para abordar con propiedad } \\
\text { el problema. } \\
\text { De él se desprenden, además, las hipótesis y las variables y tiene como funciones: delimitar el área de investigación, } \\
\text { sugerir guías, compendiar conocimientos existentes en el área a investigar, expresar proposiciones teóricas. }\end{array}$ \\
\hline Marco legal & $\begin{array}{l}\text { él se expone la normatividad vigente que rodea el proyecto. No se transcribe la norma, solo se citan algunos apartes } \\
\text { importancia para el proyecto, haciendo el comentario que corresponda. }\end{array}$ \\
\hline Metodo & $\begin{array}{l}\text { Constituye la médula del proyecto. Contempla estrategias, procedimientos, actividades, medios requeridos para cumplir } \\
\text { los objetivos propuestos y dar respuesta al problema planteado. } \\
\text { El diseño metodológico, muestral y estadístico constituye la estructura sistemática para el análisis de la información, } \\
\text { nos lleva a interpretar los resultados en función del problema que se investiga y de los planteamientos teóricos del } \\
\text { mismo diseño. } \\
\text { La forma de organización de las actividades metodológicas dependen del tipo de proyecto y puede variar sustancialmente } \\
\text { de un proyecto a otro. }\end{array}$ \\
\hline $\begin{array}{l}\text { Tipo de } \\
\text { investigación }\end{array}$ & $\begin{array}{l}\text { El tipo de investigación o de estudio debe ser acorde con la investigación a realizar, ya que cada una de estas exige una } \\
\text { estrategia diferente para su tratamiento metodológico. Según el objeto de estudio la investigación puede ser: básica } \\
\text { aplicada, clínica, analítica y de campo. } \\
\text { Según el nivel de medición y análisis de la información: cualitativa, cuantitativa, descriptiva, explicativa, inferencial y } \\
\text { predictiva. Según las variables: simple, amplia, semi-experimental y experimental. }\end{array}$ \\
\hline & $\begin{array}{l}\text { a población es la totalidad de elementos del fenómeno a estudiar. Sus unidades poseen una característica común, la } \\
\text { ual se estudia y da origen a los datos de la investigación y si de esa seleccionamos algunos elementos con la intención } \\
\text { e averiguar algo sobre dicha población de la cual están tomados, nos referimos a la muestra, esperando que lo que } \\
\text { veriguamos en la muestra sea cierto para la población como conjunto. }\end{array}$ \\
\hline $\begin{array}{l}\text { Técnicas de } \\
\text { recolección de } \\
\text { información }\end{array}$ & $\begin{array}{l}\text { Se explica el procedimiento, lugar y condiciones de la recolección de datos, depende del tipo de investigación y del } \\
\text { problema planteado. } \\
\text { Se indica si la investigación va a ser con base en lecturas, encuestas, análisis, de documentos y observación directa de } \\
\text { los hechos. También incluyen los pasos que se darán y las instrucciones para quien deba recoger los datos. }\end{array}$ \\
\hline $\begin{array}{l}\text { Análisis de la } \\
\text { información } \\
\text { recolectada }\end{array}$ & La información recolectada se analiza, compara y presenta para confirmar o rechazar las hipótesis planteadas. \\
\hline $\begin{array}{l}\text { Cronograma } \\
\text { de actividades }\end{array}$ & $\begin{array}{l}\text { Comprende la descripción de las actividades en relación con el tiempo en el cual se van a desarrollar. De acuerdo con } \\
\text { los recursos, el tiempo total y el equipo humano con que se cuenta, se calcula para cada una de ellas el tiempo en el cual } \\
\text { se van a llevar a cabo. } \\
\text { Para su presentación se usan diferentes tipos de diagramas como el de Gantt, el de PERT y el CPM. }\end{array}$ \\
\hline & $\begin{array}{l}\text { Expresan los logros del proyecto, los cuales deben ser concretos y verificables, nos permiten reconocer hasta qué punto } \\
\text { podemos confirmar o refutar las hipótesis. } \\
\text { Los resultados pueden estar representados en forma de nuevo conocimiento, información, bienes, servicios, la } \\
\text { contribución a la formación como investigadores o al fortalecimiento del grupo de investigación, consolidación de } \\
\text { redes, avance en el desarrollo de líneas de investigación, etc. }\end{array}$ \\
\hline Impacto & $\begin{array}{l}\text { Se refiere al alcance que tiene la investigación en los ámbitos científico, tecnológico, ambiental, económico o social. } \\
\text { Es el beneficio potencial del proyecto y solo será real en la medida en que sea utilizado por la población implicada y se } \\
\text { defienda suficientemente. }\end{array}$ \\
\hline
\end{tabular}


Continuación Tabla 11

\begin{tabular}{|c|c|}
\hline $\begin{array}{l}\text { Estrategias de } \\
\text { comunicación }\end{array}$ & $\begin{array}{l}\text { Es la forma cómo se dan a conocer los resultados de la investigación. } \\
\text { Se determinan las acciones mediante las cuales se ha de divulgar y socializar la información producida en la } \\
\text { investigación, para lo cual se requiere tener comunicación permanente con la población tomada y con las entidades que } \\
\text { tengan relación con el proyecto y se comprometan a algunos eventos en los que pretenda participar, con el fin de llegar } \\
\text { a diferentes clases de comunidades. }\end{array}$ \\
\hline Presupuesto & $\begin{array}{l}\text { Se hace para realizar un cálculo anticipado de los ingresos y gastos en relación con el proyecto, y su es asignar las } \\
\text { cantidades necesarias para el desarrollo de las actividades contempladas en sus rubros. } \\
\text { Se deben tener en cuenta dos aspectos: } \\
\text { 1. Fuentes de financiación del proyecto: al saber cuánto cuesta el proyecto, cómo se van a obtener los recursos } \\
\text { económicos (si los hay en la universidad o es necesario obtenerlos de algunas entidades). } \\
\text { 2. Rubros de gastos del proyecto: realizando un cálculo anticipado de los ingresos con relación detallada de las } \\
\text { actividades que generan gastos. }\end{array}$ \\
\hline Conclusiones & Se sintetizará el resultado de la investigación. \\
\hline Bibliografía & $\begin{array}{l}\text { Comprende la documentación que se consultó para la formulación del proyecto. } \\
\text { Las diferentes publicaciones utilizadas o que han servido de apoyo o fundamento para la formulación del proyecto se } \\
\text { registran o relacionan en forma alfabética. }\end{array}$ \\
\hline Anexos & $\begin{array}{l}\text { Se debe hacer referencia a ellos en el contenido o cuerpo de la obra. Están compuestos por gráficas, mapas, cuadros, } \\
\text { estadísticas, documentos y todo tipo de ilustraciones que se crea conveniente insertar en el trabajo, pero separados de la } \\
\text { obra. Deben aparecer en el mismo orden en que han sido citados, indicando su número y correspondiente título. }\end{array}$ \\
\hline
\end{tabular}

Fuente: Elaboración propia con información recabada de Castillo (2004)

Tomado y adaptado del libro de Mauricio Castillo: Guía para la formulación de proyectos

La Tabla 11 es en sí una propuesta que busca en los estudiantes y futuros tecnólogos planean la facilidad de la investigación y el interés para iniciarse en los procesos investigativos y a proyectarse como investigadores. Trae consigo una diversidad de beneficios y alcances a quienes se inician en la investigación. En verdad cuando existe un camino a recorrer es fácil, pero cuando en los procesos investigativos no hay camino, se hace camino al investigar y, en tal caso, esta es la tarea de docentes y estudiantes que hacen parte de los programas académicos a los que se han hecho referencia.

\section{Normas de presentación de trabajos escritos}

En la elaboración, la presentación y la ejecución de proyectos de investigación formativa y posteriormente aplicada, se requiere del uso adecuado y correcto de normas que hacen más técnico y organizado el proceso.

Existen de manera básica, las normas APA e ICONTEC. La ICONTEC en su última versión y las APA, en la versión $6^{a}$, facilitan a los estudiantes de carreras tecnológicas un uso adecuado.

Las normas APA e ICONTEC tienen como objetivo evitar el plagio y respetar los derechos de autor, según la normatividad legal en campo civil. Las dos normas, así como las demás buscan organizar un protocolo formal de los procesos de escritura académica y científica como resultado de los procesos de indagación y revisión de la literatura y de la sistematización de los resultados de los procesos de investigación. 
Proyección de escritura académica y científica

Es un verdadero reto que los estudiantes de las tecnologías de Regencia en Farmacia y Gestión en Salud no solo conozcan acerca de las diferentes clases y tipos de artículos desde la enseñanza de la asignatura de Investigación, sino que además puedan escribir y publicar en las revistas clasificadas por Colciencias en: A1, A2, B, C y no indexada, según las indicaciones de la Tabla 12.

Tabla 12. Cuadro de las clases de artículos según Colciencias

\begin{tabular}{|c|l|}
\hline No. & \multicolumn{1}{|c|}{ Tipología del artículo } \\
\hline 1 & $\begin{array}{l}\text { Artículo de investigación científica y tecnológica. } \\
\text { Documento que presenta, de manera detallada, los } \\
\text { resultados originales de proyectos terminados de } \\
\text { investigación. La estructura generalmente utilizada } \\
\text { contiene cuatro apartes importantes: introducción, } \\
\text { metodología, resultados y conclusiones. }\end{array}$ \\
\hline 2 & $\begin{array}{l}\text { Artículo de reflexión. Documento que presenta resultados } \\
\text { de investigación terminada desde una perspectiva } \\
\text { analítica, interpretativa o crítica del autor, sobre un tema } \\
\text { específico, recurriendo a fuentes originales. }\end{array}$ \\
\hline 3 & $\begin{array}{l}\text { Artículo de revisión. Documento resultado de una } \\
\text { investigación terminada donde se analizan, sistematizan } \\
\text { e integran los resultados de investigaciones, publicadas o } \\
\text { no publicadas, sobre un campo en ciencia o tecnología, } \\
\text { con el fin de dar cuenta de los avances y las tendencias } \\
\text { de desarrollo. Se caracteriza por presentar una cuidadosa } \\
\text { revisión bibliográfica de por lo menos 50 referencias. }\end{array}$ \\
\hline $\begin{array}{l}\text { Reporte de caso. Documento que presenta los resultados } \\
\text { de un estudio sobre una situación particular con el fin de } \\
\text { dar a conocer las experiencias técnicas y metodológicas } \\
\text { consideradas en un caso específico. Incluye una revisión } \\
\text { sistemática comentada de la literatura sobre casos } \\
\text { análogos. }\end{array}$ \\
\hline
\end{tabular}

Fuente: Elaboración propia

Tomado de la carta de presentación de artículos de la Revista Praxis \& Saber, de la Maestría en Educación de la Universidad Pedagógica y Tecnológica de Colombia

La Tabla 12 es, en primer lugar, informativa, pues invita a los estudiantes a leer, investigar y también escribir no solo proyectos de investigación, sino artículos de revisión, reflexión e investigación como una forma de dar a conocer la sistematización de los resultados. Una vez se adelanten los procesos de investigación y se consoliden los procesos de escritura, los trabajos se presentan a las convocatorias de las diferentes revistas según la base institucional y de Colciencias, en las cuales los Comités Editoriales permiten que inicie un proceso de evaluación y posible publicación según las políticas de cada revista y área de visibilidad.

La universidad, a través de la Dirección de Investigaciones, ha reconocido muchos grupos de investigación de los centros de investigación de cada Facultad, y en cada uno de estos grupos hay semilleros de investigación. La Facultad de Estudios a Distancia cuenta con cuatro grupos de investigación: TICA, categorizado en C; SIEK, en B; GIGAS, en C y CIMA reconocido a nivel institucional. El grupo GIGAS hace parte de la Escuela de Ciencias Administrativas y Económicas, a la cual se encuentran adscritos los programas de Regencia en Farmacia y Gestión en Salud. Este grupo está categorizado en C por Colciencias, y desde la coordinación se ha propuesto con la razón y el corazón la importancia de que docentes y estudiantes formen parte como investigadores, co-investigadores y semilleros de investigación y que se vea reflejado la visibilidad de la investigación con la publicación de artículos en revistas indexadas y de alto impacto nacional e internacional. 
La investigación en el entorno académico, laboral y profesional

Reyes et al. (2005) plantearon una reflexión respecto a si vale la pena formar ingenieros o tecnólogos, los mismos autores presentan un paralelo entre Colombia y Europa al respecto y afirman: "se debe formar ingenieros en largas y costosas carreras de 5 años o más, o por el contrario, se debe buscar una educación basada en ciclos propedéuticos en que los técnicos laborales, los tecnólogos y posteriormente ingenieros, puedan asegurar una vinculación laboral y productiva desde el primer año" (p.167). En este caso le apostamos a formar tecnólogos pero con la debida fortificación de habilidades y competencias para el desenvolvimiento en su vida laboral y de la investigación. A pesar de ello, se infiere aún le falta de más tiempo, compromiso, políticas y estrategias para fomentarla y construirla.

El entorno laboral requiere de tecnólogos idóneos y capaces de realizar a cabalidad su ejercicio laboral. En consecuencia, se debe despertar en ellos "un sentido por la curiosidad y el asombro que está en la base de cualquier trabajo investigativo" (Pineda, 2011, p.45), mas no solo en el trabajo investigativo sino en el desempeño laboral, ya que se requiere de personas creativas e idóneas en el ejercicio de su trabajo, así como compromiso con la investigación formativa y aplicada.

Durante la formación académica de los tecnólogos en Regencia en Farmacia y Gestión en
Salud se ha de inculcar, por tanto, la formación de investigación para responder a la realidad y el contexto profesional y laboral. Además, es pertinente que los estudiantes se formen como sujetos críticos, creativos, autónomos y que valoren la actualización de sus competencias, de manera que puedan aprender permanentemente. La formación en investigación debe contribuir a ello desde la perspectiva del aprendizaje estratégico que propone Feo (2015):

El aprendizaje estratégico desde sus inicios fue considerado como una tendencia emergente en los años 70 del siglo pasado y se empleó como epicentro de principios para generar propuestas de intervención pedagógicas en diversas áreas curriculares; paulatinamente, junto al avance científico, el uso de las tecnologías de la información y comunicación (TIC) en el ámbito educativo y la consolidación de la metacognición en los preludios educativos fortificaron los principios del aprendizaje estratégico y sus premisas, enriqueciendo la praxis diaria centrada en el diálogo de saberes, lo que trajo como resultado la estabilidad de este constructo para ser considerado en el siglo XXI un enfoque; en consecuencia, germinaron acciones educativas como alternativas de proposiciones dentro de los sistemas educativos latinos. (p.222)

El propósito del aprendizaje estratégico es promover el "aprender permanentemente". Para ello se requiere el compromiso del sujeto y de renovados sistemas educativos. En este sentido, la Universidad Pedagógica y Tecnológica se encuentra en constante transformación de los 
contenidos curriculares, de acuerdo con las transformaciones sociales, políticas, económicas y del contexto, sin perder de vista la formación integral de los educandos, que también serán futuros agentes de cambio en la sociedad.

\section{Conclusiones}

La importancia de la formación de la investigación en los tecnólogos tiene aspectos relevantes como el conocerse a sí mismo y hacer una verdadera reflexión sobre el contexto para formular proyectos pertinentes. De igual forma, ello propicia la escritura y publicación de artículos de investigación en revistas indexadas y especializadas con el fin de dar a conocer los resultados y la sistematización de significativos procesos.

Desde que los estudiantes inician su de formación tecnológica han de recibir una verdadera proyección sobre la forma de participar en procesos de investigación y la integración a semilleros y grupos de investigación, asumiéndolos no solo como una posibilidad de grado, sino como una forma de acceder a becas de investigación promovidas por la Unidad de Política Social de la universidad y, sobre todo, como una opción de vida. Es clave que ellos entiendan la necesidad de formarse para iniciar procesos de investigación y transformar verdaderas problemáticas que se presentan en la vida cotidiana de la Gestión en Salud y de la Regencia en Farmacia.

Actualmente, la investigación se adentra en todas las ramas del saber y es aplicable a las exigencias de la sociedad. La formación de tecnólogos en Regencia en Farmacia y Gestión en Salud no es la excepción al respecto, y ello debe fortalecer su formación académica y su posterior ejercicio profesional y laboral.

Es importante resaltar los avances investigativos en la formación de las tecnologías de Regencia en Farmacia y Gestión en Salud, desde donde se evidencian progresos en problemáticas relacionadas con la educación a distancia. No obstante, aún se ha de fortalecer la formación en investigación en los docentes y a los estudiantes. En este sentido, se requiere que a la hora de la asignación académica se tenga en cuenta la idoneidad de quienes orienten esta asignatura. Además estos docentes deben hacer parte de un grupo de investigación, promover la formación de semilleros de investigación, elaborar $y$ presentar proyectos de investigación $\mathrm{y}$, desde luego, escribir y publicar artículos y otros tipos de textos que sean en sí resultados de procesos de investigación.

Es la hora de encaminar a los educandos de carreras tecnológicas a que hagan parte de semilleros y grupos de investigación, con el propósito de generar en ellos conocimiento a partir de realidades existentes en su contexto. Se requiere que los estudiantes y futuros tecnólogos tengan manejo de competencias, habilidades y herramientas investigativas con el fin de fortalecer su proceso académico, profesional y laboral.

Este artículo pretende destacar la importancia y la necesidad de una formación en investigación 
en la vida de tecnólogos de Regencia en Farmacia y Gestión en Salud, que ellos se constituyan en verdaderos investigadores, así como en éticos e idóneos profesionales. De manera particular, se debe generar y desarrollar en los tecnólogos un espíritu y unas fortalezas y competencias investigativas, que los hagan capaces de desarrollar procesos, proyectos y artículos de investigación.

En realidad, aún falta una idónea formación de los docentes en investigación y de ellos hacia a los estudiantes, para que así estos se motiven y se apasionen por hacer parte de semilleros, grupos y procesos investigativos. Que se atrevan, en últimas, a formular y presentar proyectos de investigación para ejecutarlos y que, a través de sus directores de grupos de investigación, puedan acceder a recursos y a convocatorias de las universidades, a nivel regional, nacional e internacional.

Hoy en día, las bases de investigación y formulación de proyectos en los estudiantes de educación superior en la modalidad a distancia y en las carreras en el nivel de tecnologías son totalmente necesarias para fortalecer los principios de investigación formativa y propiamente científica, que no han de ser ajenos al contexto $\mathrm{y}$, así, garantizar la producción de nuevo y eficaz conocimiento.

\section{Referencias}

Acuerdo No. 050 de 2008. Por el cual se establecen los criterios para la implementación del Sistema de Créditos y se definen

\section{las Áreas de Estructuración Curricular}

de los Programas de Pregrado Presenciales, en la Universidad Pedagógica y Tecnológica de Colombia.

Acuerdo No. 086 de 2009. Por el cual se reglamenta parcialmente el acuerdo 050 de 2008.

Álvarez, A. et al. (1.983). Guía de investigación. $1^{\mathrm{a}}$ ed. Bogotá Colombia: Ediciones Usta.

Basave, F. (2007). Educación a distancia en el nivel superior. $1^{\mathrm{a}}$ ed. México: Editorial Trillas.

Castillo, M. (2004). Guía para la Formulación de Proyectos de investigación. $1^{\mathrm{a}}$ ed. Bogotá D. C. Colombia: Editorial Magisterio.

Corrales, M. I. (2008). Metodología de la formación abierta y a distancia. $1^{\mathrm{a}}$ ed. Balderas México: Editorial Limusa, S. A.

Cortés, R. (s.f.). La educación a distancia y el estudio independiente, consultado en línea: http://red.ilce.edu.mx/sitios/ revista/e_formadores_oto_09/articulos/ Angeles_Cortes.pdf

Cerda, G. H. (2000). La evaluación como experiencia total. Logros-objetivosprocesos, competencias y desempeños. $1^{\mathrm{a}}$ ed. Bogotá: Editorial Magisterio.

Decreto 2412 agosto 19 de 1982, “por el cual se reglamenta, dirige e inspecciona la Educación Abierta y a Distancia y se crea el consejo de Educación Abierta y a Distancia".

De la Torre, Z. F. (2009). 12 Lecciones de pedagogía, educación y didáctica. $1^{\mathrm{a}}$ ed. México: Editorial Alfaomega. 
Castillo, M. (2.004). Guía para la formulación de proyectos de investigación. $1^{\mathrm{a}}$ ed. Bogotá D. C. Colombia: Editorial Magisterio.

Decreto 2566 de 10 de septiembre (2003). Por la cual se establecen las condiciones mínimas de calidad y demás requisitos para el ofrecimiento y desarrollo de programas académicos de educación superior. $1^{\mathrm{a}}$ ed. Bogotá D. C. Colombia: Editorial Unión.

Decreto 1358 de 11 de julio (1974). Por el cual se dictan normas sobre la educación superior. http://www.mineducacion.gov. co/1621/article-104755.html

Decreto 882 de 10 de mayo (1976). Por el cual se reglamenta el decreto extraordinario $N^{\circ}$. 89 de 1976. http://www.mineducacion. gov.co/1621/article-102590.html

Decreto 2667 de 17 de diciembre (1976). Por el cual se reglamentan las carreras tecnológicas. http://www.mineducacion.gov. co/1621/article-102595.html

Decreto No. 129520 de abril de 2010. Por el cual se reglamenta el registro calificado de que trata la Ley 1188 de 2008 y la oferta y desarrollo de programas académicos de educación superior. http://www.mineducacion.gov.co/1621/articles- 229430 archivo_pdf_decreto1295.pdf

Doyle, A. (2005). Las aventuras de Sherlock Holmes. $1^{\mathrm{a}}$ ed. Bogotá: Editorial Unión.

Floréz, R. (1999). Evaluación pedagógica y cognición. $1^{\mathrm{a}}$ ed. Bogotá D. C. Colombia: Editorial McGrawHill.

Feo, R. (2015). Epistemología y práctica de la investigación sobre el aprendizaje estratégico en América Latina. Revista Educación y Humanismo, 17(29), 220-235. http://dx.doi.org/10.17081/ eduhum.17.29.1254

Ley 30 de 29 de diciembre (1992). Por la cual se organiza el servicio público de la Educación Superior. $1^{\mathrm{a}}$ ed. Bogotá D. C. Colombia: Ediciones Momo.

Ley 749 de 19 de julio (2002). Por la cual se organiza el servicio público de la educación superior en las modalidades de formación técnica profesional y tecnológica. $1^{\mathrm{a}}$ ed. Bogotá D. C. Colombia: Editorial Unión.

Munch, L. et al. (2.009). Métodos y técnicas de investigación. $4^{\mathrm{a}}$ ed. México: Ed Trillas.

Pineda. A. (2008). La construcción del oficio del investigador: una perspectiva Sherlockiana. $1^{\mathrm{a}}$ ed. Bogotá: Editora Beta.

Pineda, A. (2011). El investigador universitario. Seminario permanente de pedagogía, Revista de la Universidad Pedagógica y Tecnológica de Colombia. Tunja. CIEFEC, 3 (1) 30 - 51

Ravelo, E. (2002) Calidad, aprendizaje y rendimiento académico en educación superior. Educación y Humanismo, Vol. 14 - No. 23 - pp. 17-36 - diciembre, 2012 - Universidad Simón Bolívar - Barranquilla, Colombia - ISSN: 0124-2121 http:// portal.unisimonbolivar.edu.co:82/rdigital/ educacion/index.php/educacion

Reyes, F.21.23 de sepriembre de 2005). ¿Formar ingenieros o tecnólogos? El impacto de las reformas de la educación superior en la formación de ingenieros. $X X V$ Reunión 
Nacional de Facultades de Ingeniería. Resolución Na. 36 de 2010 Por la cual se adopta Cartagena de Indias. y reglamenta las modalidades de trabajo Resolución No. 58 de 2009 Por la cual se de grado para los estudiantes de los modifica la resolución 40 de 2008.

http://www.uptc.edu.co/export/sites/default/ secretaria_general/consejo_academico/ resoluciones_2009/res_058_2009_ modif_res40.pdf programas de pregrado ofrecidos por la facultad de estudios a distancia de la Universidad Pedagógica Tecnológica de Colombia. Consultada en:http://www. uptc.edu.co/export/sites/default/secretaria_general/consejo_academico/resoluciones_2010/res_36_2010.pdf 